\title{
Estimation simple de la surface foliaire de plantes de maïs en croissance
}

\author{
F Ruget *, R Bonhomme, M Chartier \\ Unité de recherche en bioclimatologie, Inra, F-78850 Thiverval-Grignon, France
}

(Reçu le 30 novembre 1995; accepté le 16 octobre 1996)

\begin{abstract}
Summary - A simplified method for estimating the leaf area growth of field-grown maize from a reduced number of measurements $A$ simplified method for leaf area estimation in corn is described. It is shown that during the first half of leaf growth period, growing leaves form the major part of total leaf area. The method uses the number of growing leaves as well as the number and size of full grown leaves. Estimated leaf area and intercepted light are compared with actual leaf measurements (table I) and derived intercepted light estimations (tables I and II). The method produces adequate estimations at flowering but overestimates intercepted light during the first half of leaf growth.
\end{abstract}

\section{leaf area / maize / growing leaves}

Résumé - Cet article présente une méthode d'estimation de la surface foliaire d'une plante de maïs pendant la mise en place des feuilles. II montre que, pendant la première moitié de cette période, les feuilles en croissance forment la majeure partie de la surface foliaire totale. La méthode d'estimation de surface proposée ne nécessite, en plus de la mesure de leur taille finale, que des comptages de feuilles pendant leur mise en place. La comparaison à des mesures de référence montre que cette méthode donne des résultats satisfaisants en matière d'estimation de surface et surtout de rayonnement capté à la floraison, mais elle surestime légèrement le rayonnement capté pendant la première moitié de la mise en place des feuilles.

surface foliaire / maïs / feuilles en croissance / feuilles ligulées / feuilles non ligulées

\section{INTRODUCTION}

La plupart des modèles de fonctionnement de cultures considèrent maintenant que la production de matière sèche du couvert peut être estimée à partir du rayonnement solaire utile à la photosynthèse qu'il capte (Monteith, 1972). Or la proportion de rayonnement intercepté est estimée à partir de la surface foliaire qui est donc une variable importante pour la détermination de la production d'une culture.

Le but de cet article est de présenter une méthode d'estimation de la surface foliaire par plante pendant la mise en place des feuilles, à 
partir de mesures faciles à réaliser au champ et fiables. Ce n'est ni une modélisation de la surface foliaire maximale, ni une modélisation de la surface finale des feuilles ou de leur répartition en hauteur dans le couvert.

Chez les graminées, et le maïs en particulier, la feuille est constituée d'un limbe et d'une gaine qui entoure la tige et recouvre parfois partiellement celle de la feuille suivante. La ligule marque la séparation entre limbe et gaine. Le limbe est la partie qui assure l'essentiel de l'interception du rayonnement et de la photosynthèse. Nous le désignerons simplement par feuille dans la suite du texte.

La surface foliaire d'une plante est la somme des surfaces de chacune de ses feuilles. Les feuilles ont fini leur croissance lorsque leur ligule est apparue : celles qui sont ligulées ont donc une surface fixée, tandis que celles qui sont en croissance ont une surface qui évolue au fur et à mesure de leur étalement. Elles forment le "cornet de feuilles".

Les méthodes de mesure et d'estimation des surfaces des feuilles ligulées et non ligulées sont différentes. C'est pourquoi nous les traiterons séparément. Dans les deux cas, nous nous doterons d'une mesure de référence, puis nous proposerons plusieurs méthodes d'estimation que nous comparerons.

La mesure directe non destructive de la surface des feuilles ligulées est possible à l'aide de planimètres de terrain, mais leur emploi cause souvent des dommages à la culture (casse des feuilles). La précision de la mesure, en conditions réelles d'utilisation, avec des feuilles adultes dont la surface n'est pas très plane, est assez faible. Celle des feuilles en croissance n'est pas possible, en raison de leur disposition en cornet et de leur emboîtement.

La surface des feuilles ligulées peut être estimée à partir de mensurations simples, en raison de leur forme et de leur dimension. La forme des feuilles entièrement développées a été décrite par des expressions analytiques reliant longueur et largeur (arcs de parabole: Bonhomme et Varlet-Grancher, 1978 ; Prévot et al, 1991 ; arcs de sinusoïdes : Edmeades et Daynard, 1979 ; Sanderson et al, 1981), qui, par intégration, permettent de calculer leur surface. Au contraire, l'estimation de la surface des feuilles en croissance est rarement abordée séparément (Dwyer et Stewart, 1986 ; Muchow et Carberry, 1989) car elle nécessite des mesures supplémentaires. L'objet de ce travail est d'une part, l'estimation de la surface foliaire visible hors du cornet et l'inter- ception de rayonnement lui correspondant, d'autre part, la mise au point d'une méthode de mesures utilisables au champ de façon systématique.

Estimer l'évolution de la surface des feuilles à partir de comptages de feuilles pendant leur apparition et de mesures à la fin de leur croissance évite de toucher les feuilles pendant leur croissance donc minimise les stress liés à la manipulation des plantes ou des feuilles, tels que les a mis en évidence Beardsell (1977) sur maïs : des mesures quotidiennes de feuilles réduisaient significativement les poids de tige et les hauteurs de plante.

Enfin, la simplification et l'allégement des mesures permettent également de multiplier les situations contrôlées.

Nous évaluerons les incertitudes liées à l'utilisation, dans un modèle de fonctionnement de culture, de plusieurs méthodes d'estimation de la surface foliaire d'une plante de maïs en croissance, en les comparant à la mesure de référence, au pas de temps journalier:

- une méthode employée dans la littérature (Muchow et al, 1990),

- un ensemble de méthodes d'estimation de la surface foliaire d'une plante en croissance, qui s'appuient sur des hypothèses attribuant une proportion de longueur ou de surface visible à chaque feuille du cornet. L'effet de ces hypothèses sur la qualité de l'estimation de la surface foliaire et du rayonnement intercepté sera jugé au travers de critères statistiques adaptés.

\section{MATÉRIEL ET MÉTHODES}

Les mesures ont porté sur deux lots de dix plantes contiguës, situées sur des rangs voisins à l'intérieur d'une parcelle de 1 ha, cultivée à Grignon $\left(48^{\circ} \mathrm{N}, 1^{\circ} \mathrm{E}\right.$, altitude $100 \mathrm{~m}$ ) en 1990 dans des conditions agronomiques classiques pour la région (semis le 4 mai, j124). Parmi ces 20 plantes, un échantillon de six plantes a été utilisé pour les comparaisons entre méthodes et les évolutions moyennes de surface. Le génotype employé est un hybride simple, Dea, dont le nombre total de feuilles est voisin de 16. Un trait de couleur différente a été tracé chaque jour à la base de la partie visible de chaque feuille en croissance. À la fin de l'émission des feuilles, les plantes ont été prélevées. Les hauteurs et largeurs de chaque trapèze (croissance journalière) ont été mesurées ainsi que les dimensions maximales des feuilles.

Deux autres lots de mesures sont utilisés en complément: il s'agit des dimensions de feuilles de l'expérimentation décrite par Chartier et al (1971) et 
utilisées partiellement par Bonhomme et Varlet Grancher (1978), réalisées à La Minière $\left(48^{\circ} \mathrm{N}, 1^{\circ} \mathrm{E}\right)$ avec la variété $F 7^{\star} E p 1$ et de celles de Prévot et al (1991), réalisées à Avignon ( $\left.44^{\circ} \mathrm{N}, 5^{\circ} \mathrm{E}\right)$, avec la variété Dea.

\section{Méthode de mesure de la surface des feuilles ligulées et non ligulées en croissance (méthode de référence)}

Les traits marqués sur les feuilles pendant leur croissance fournissent une mesure de leur accroissement journalier en longueur et en surface. En cumulant les surfaces des éléments trapézoïdaux du sommet à la base de la feuille, on obtient sa surface maximale (méthode $\mathrm{SL}_{\text {réf }}$ pour les feuilles ligulées). Si on cumule les surfaces élémentaires du sommet à la base de la partie visible de la feuille à une date donnée, on obtient sa surface visible à cette date (méthode MO pour les feuilles non ligulées). Par somme pour l'ensemble des feuilles, on a accès aux surfaces «ligulée» et «non ligulée» de la plante.

\section{Estimations de la surface des feuilles ligulées}

\section{Calcul classique}

La surface maximale des feuilles (SL) est calculée classiquement par le produit des longueur et largeur maximales $\left(L_{\text {max }}\right.$ et $\left.I_{\text {max }}\right)$ affecté du coefficient 0,75 (Bonhomme et al, 1982, par exemple).

\section{Calcul prenant en compte la forme de la feuille}

Les lois de forme établies par Bonhomme et Varlet Grancher (1978) permettent de calculer la surface d'une feuille en décrivant la variation de la largeur en fonction de l'abscisse le long de la nervure centrale. La loi de forme qui relie longueur et largeur peut s'écrire :

$$
\frac{1}{I_{\max }}=a+b \frac{x}{\mathrm{~L}_{\max }}+c\left(\frac{x}{\mathrm{~L}_{\max }}\right)^{2}
$$

où / est la largeur de la feuille à la distance $x$ de sa base. La fraction de surface foliaire apparue, pour une longueur visible $v$ est :

$$
\frac{\mathrm{S}}{S_{\max }}=\frac{1}{S_{\max }} \int_{L_{\max }-v}^{L_{\max }} 1 \cdot d x
$$

elle peut être calculée en intégrant l'expression précédente:

$$
\begin{aligned}
\frac{\mathrm{S}}{\mathrm{S}_{\max }} & =\frac{\left(\mathrm{I}_{\text {max }} \cdot \mathrm{L}_{\text {max }}\right)}{\mathrm{S}_{\max }} \cdot\left\{a\left(1-\frac{\mathrm{L}_{\max }-\mathrm{v}}{\mathrm{L}_{\max }}\right)+\frac{b}{2}\left[1-\left(\frac{\mathrm{L}_{\text {max }}-\mathrm{V}}{\mathrm{L}_{\max }}\right)^{2}\right]\right. \\
& \left.+\frac{c}{3}\left[1-\left(\frac{\mathrm{L}_{\max }-\mathrm{v}}{\mathrm{L}_{\max }}\right)^{3}\right]\right\}
\end{aligned}
$$

Lorsque $x$ égale 0 , la longueur de la partie visible de la feuille est alors égale à $L_{\text {max }}$. À partir de l'équation [3], on peut exprimer la surface maximale de la feuille en fonction de $L_{\text {max }}$ et $I_{\text {max }}$ :

$$
\mathrm{S}_{\max }=\mathrm{L}_{\max } \cdot \mathrm{I}_{\max } \cdot\left(a+\frac{b}{2}+\frac{c}{3}\right)
$$

Les trois paramètres $a, b$ et $c$ de l'équation [1] sont liés entre eux du fait que la largeur de la feuille est nulle à son extrémité, soit que $l=0$ pour $x=\mathrm{L}_{\text {max }}$, soit

$$
a+b+c=0
$$

et que, lorsque la largeur est maximale, sa dérivée est nulle, ce qui conduit à l'équation :

$$
b+2 c L_{l m}=0
$$

où $L_{/ m}$ est l'abscisse du point de largeur maximale, ce qui, en reportant $L_{l m}$ comme valeur de $x$ dans l'équation [1], donne une seconde relation entre $a, b$ et $c$ :

$$
\frac{1}{I_{\max }}=a-\frac{b^{2}}{4 c}=1
$$

À l'aide des équations [5] et [7], on obtient donc des expressions de $a$ et $b$ en fonction de $c$

$$
\begin{gathered}
b=-2(\sqrt{-c}+c) \\
a=2 \sqrt{-c}+c
\end{gathered}
$$

donc une expression de $S^{*}$, le rapport entre surface et produit longueur-largeur, en fonction d'un seul des trois paramètres :

$$
\frac{\mathrm{S}_{\max }}{\mathrm{L}_{\max } \cdot \mathrm{I}_{\max }}=\mathrm{S}^{\star}=\frac{c}{3}+\sqrt{-c}
$$

Le coefficient $a$, qui est le rapport entre la largeur à l'origine et la largeur maximale, est compris entre 0 (largeur maximale au milieu et nulle à la base) et 1 (largeur maximale à la base), $c$ est compris entre -1 et -4 (Prévot et al, 1991), ce qui donne, pour $S^{\star}$, des valeurs de 0,66 aux bornes et 0,75 au maximum (qui est obtenu pour $c=-2,25$ ). Les valeurs les plus fréquentes de $c$ sont situées entre $-1,5$ et $-2,25$ pour des feuilles adultes, ce qui conduit à des valeurs de $S^{*}$ compris respectivement entre 0,72 et 0,75 (Prévot et al, 1991), donc des coefficients peu variables. Ces valeurs théoriques seront comparées à celles obtenues à partir de régressions entre surface de référence (SL réf, mesurée par éléments) et dimensions maximales.

\section{Estimation au planimètre}

La surface des feuilles a aussi été planimétrée (SL plani) avec un appareil de laboratoire LI 3100 (LICOR, Inc, Box 4425, Lincoln, NE 68504, États-Unis) avec convoyeur ; la résolution de cet appareil est le 
$\mathrm{mm}^{2}$; la précision théorique de sa mesure est $\pm 1 \%$ pour des surfaces supérieures à $10 \mathrm{~cm}^{2}$. Le planimètre est étalonné avant chaque série de mesures à l'aide d'une surface étalon de $50 \mathrm{~cm}^{2}$. Le principe de la mesure est la proportion de rayonnement arrêtée par la feuille pendant qu'elle passe entre une barrette de photoémetteurs et une barrette de photorécepteurs. La feuille est conduite entre ces barrettes en étant pincée entre deux bandes de polyéthylène tranparent. La bonne précision des mesures dépend de la planéité de la feuille, de la régularité d'avancement et, évidemment de la bonne turgescence de la feuille et de l'absence de superposition de certaines parties de feuilles.

\section{Estimations de la surface des feuilles en croissance}

La méthode (M1), présentée par Muchow et Carberry (1989) et utilisée par Muchow et al (1990), fait l'hypothèse que la surface de l'ensemble des feuilles en croissance correspond à la surface maximale des deux feuilles en croissance les plus âgées (les prochaines ligulées). Elle utilise le nombre de feuilles ligulées et la surface maximale des feuilles.

Les nouvelles méthodes proposées s'appuient sur la définition d'une fraction de surface apparue pour chacune des feuilles visibles. L'hypothèse est que, pour une date donnée, la proportion de surface (M2) ou de longueur (M3) visible d'une feuille diminue linéairement de la dernière feuille ligulée à la dernière feuille visible.

- méthode M2 : l'attribution ou non d'une surface à la dernière feuille visible conduit à deux sous-méthodes (M2a et M2b) :

$$
\begin{aligned}
& \frac{S}{S_{\max }}=\frac{(N F V+1-N F)}{(N F V+1-N F L)} \\
& \frac{S}{S_{\max }}=\frac{(N F V-N F)}{(N F V-N F L)}
\end{aligned}
$$

où NF est le numéro de la feuille dont on estime la surface ; NFL, le numéro de la dernière feuille ligulée ; NFV, le numéro de la dernière feuille visible ;

- méthode M3 : la fraction de longueur émise de chaque feuille dépend de son numéro dans le cornet rapporté au nombre de feuilles de celui-ci :

$$
\frac{v}{L_{\max }}=\frac{N F V+1-N F}{N F V+1-N F L}
$$

et la surface foliaire visible est calculée en reportant l'expression de $v / L_{\max }$ dans l'équation [3].

Ces méthodes utilisent toutes un suivi : le comptage du nombre de feuilles ligulées et visibles chaque jour (le nombre de feuilles en croissance étant la différence), et une mesure finale : la mesure des longueur et largeur maximales de chaque feuille (respectivement
$L_{\text {max }}$ et $I_{\text {max }}$ ) après expansion complète et avant sénescence. Les estimations de surface ne peuvent être faites qu'a posteriori, lorsque les feuilles concernées sont ligulées.

\section{Estimation du rayonnement capté par une culture}

Le calcul de rayonnement intercepté par le couvert est fait en utilisant l'équation

$$
\varepsilon_{i}=\left(\mid-e^{(-k * \mid F)}\right)
$$

qui relie l'efficience d'interception $\varepsilon_{i}$ à l'indice foliaire (IF). Ce dernier est calculé en multipliant la surface foliaire par plante (en mètre carré) par la densité moyenne de la culture (neuf plantes par mètre carré, ici). Le coefficient d'extinction $k$ dépend essentiellement du port des feuilles et est égal à 0,7 pour le maïs (Bonhomme et al, 1982).

\section{RÉSULTATS et DISCUSSION}

\section{Surface maximale des feuilles}

\section{Comparaison des estimations à la mesure}

La figure 1 représente la surface maximale des feuilles mesurée au planimètre $\left(S L_{\text {plani }}\right)$ et la surface estimée par le produit des dimensions de la feuille (SL) en fonction de la mesure de référence $\left(S L_{\text {réf }}\right)$. II apparaît une légère sous-estimation systématique des mesures au planimètre ; la régression obtenue sur les feuilles pour lesquelles les mesures complètes ont été possibles $(n=39)$ est :

$S L_{\text {plani }}=34,04+0,792 \cdot S L_{\text {réf }} \quad$ avec $r^{2}=0,902$

On obtient une meilleure relation entre $\mathrm{SL}_{\text {réf }}$ et $\mathrm{SL}\left(\mathrm{L}_{\max } \bullet \mathrm{I}_{\max } \bullet 0,75\right)$ :

$$
\mathrm{SL}=42,26+0,921 \cdot \mathrm{SL}_{\text {réf }} \quad \text { avec } r^{2}=0,969
$$

La surface foliaire d'une feuille ligulée de maïs peut donc être estimée avec une meilleure précision par la mesure de ses longueur et largeur maximales que par l'emploi d'un planimètre électronique, même de grande précision comme celui utilisé ici. La bonne turgescence de la feuille est indispensable. Le gaufrage de la feuille et surtout l'épaisseur de la nervure peuvent provoquer des superpositions de certaines parties de feuilles, qui causent des erreurs de mesure. 


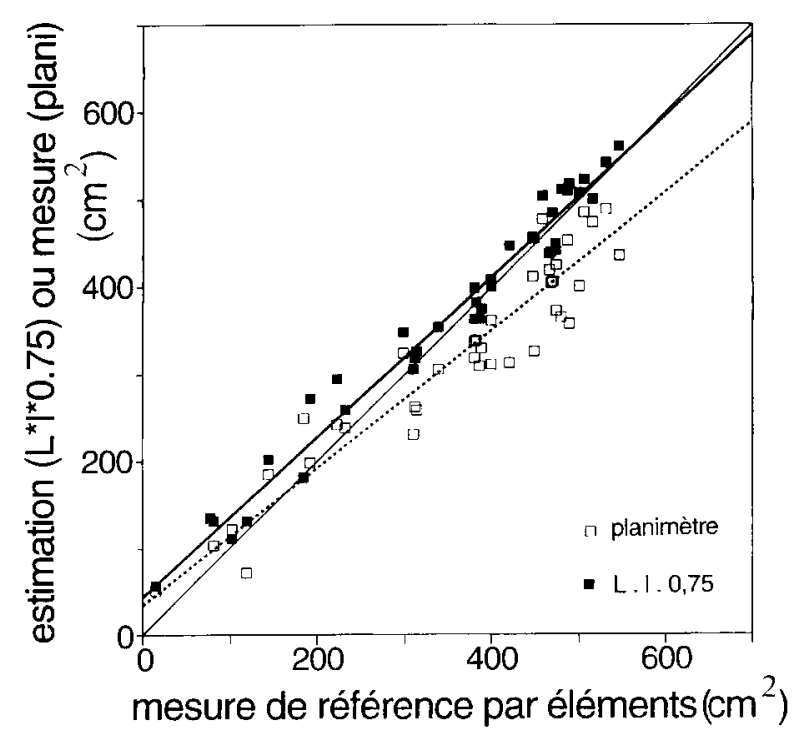

Fig 1. Surfaces des feuilles mesurées au planimètre ou estimées par le produit $0,75 \cdot L_{\max } \cdot I_{\max }$, représentées en fonction de la valeur de référence (mesure par éléments), pour tous les numéros de feuilles. La droite en trait plein fin est la première bissectrice, la droite en trait plein gras est la droite de régression des valeurs estimées par le produit $0,75 \bullet L_{\max }$ • $I_{\max }$ et la droite en pointillés, celle des mesures au planimètre.

\section{Coefficient de forme reliant dimensions et surface}

\section{Corrélation entre surface de référence et produit longueur largeur}

Le coefficient de forme $S^{\star}$, calculé comme la pente de la régression passant par l'origine entre valeurs de référence et produit longueur-largeur est égal à 0,746. Pour Prévot et al (1991), la valeur moyenne de $S^{*}$, définie comme la moyenne des coefficients de forme pondérée par la surface des feuilles,

$$
\frac{1}{n} \frac{\sum \mathrm{S}^{*} \cdot \mathrm{L}_{\max } \cdot \mathrm{I}_{\max }}{\sum \mathrm{L}_{\max } \cdot \mathrm{I}_{\max }}
$$

a la valeur de 0,749 sur un échantillon de 30 plantes, tandis qu'on obtient 0,743 sur l'échantillon de La Minière en 1971, avec des mesures au planimètre comme valeurs de référence, dans les deux cas.

\section{Régression entre surface calculée par intégration et produit longueur largeur}

L'intégration de la loi de variation de la largeur de la feuille, utilisée par la méthode $M 3$, conduit à un coefficient théorique égal à 0,75 , que ce soit avec le jeu de données obtenues sur $F 7^{\star} E p 1$ à
La Minière, pour toutes les feuilles ligulées lorsqu'on ne distingue pas leur position le long de la plante $(a=0,791, b=1,281$ et $c=-2,064)$ ou celui de Prévot et al (1991) où les distributions des valeurs les plus fréquentes de $c$ pour les feuilles ligulées s'étalent de $-2,25$ à $-1,5$.

Le coefficient habituellement employé de 0,75 est donc concordant aussi bien avec les différents jeux de mesure qu'avec les estimations théoriques liées à la forme des feuilles de graminées.

\section{Surface des feuilles non ligulées}

La figure 2 représente les proportions de surface apparues de chaque feuille en croissance, en valeurs mesurées et en valeurs estimées, pour un stade foliaire donné, défini par ses nombre de feuilles ligulées et totales visibles (ici $\mathrm{NFL}=7$, NFV = 11). Ces proportions sont les coefficients appliqués au produit $0,75 \cdot L_{\max } \bullet I_{\max }$ pour calculer les surfaces visibles de chaque feuille. La figure 2a représente les valeurs mesurées des coefficients pour des plantes situées au même stade foliaire. Elle illustre la variabilité parcourue par les coefficients au cours d'un stade. La figure $2 b$ présente les jeux de coefficients estimés par les différentes méthodes; chaque stade foliaire est représenté par un seul jeu de coefficients, l'estimation ne reproduit donc pas l'évolution des coefficients à l'intérieur d'un stade.

Les coefficients d'estimation des différents modèles sont situés au centre de la gamme des coefficients mesurés. La méthode M1 donne par construction une composition du cornet de feuilles assez différente de la réalité.

La figure $3 a$ présente le profil de surface auquel s'appliquent les coefficients de la figure 2 : la variabilité de la surface des feuilles en fonction de leur numéro, donc de leur période d'apparition, est importante. La figure $3 b$ présente l'évolution de la surface de l'ensemble des feuilles en croissance pour chacune des six plantes étudiées en valeurs mesurées : pendant la période où le cornet de feuilles est le plus important (environ quatre feuilles, cf Évolution des surfaces ligulées et non ligulées au cours du temps), les variations de la surface foliaire non ligulée sont plus importantes au cours du temps pour chaque plante qu'entre plantes.

La figure 4 représente la surface visible de chaque feuille (correspondant aux proportions de la figure 2) à trois dates (début, milieu et fin de mise en place des feuilles), et pour les différentes méthodes (mesure et estimation). Elle illustre les écarts entre les surfaces estimées par les diffé- 


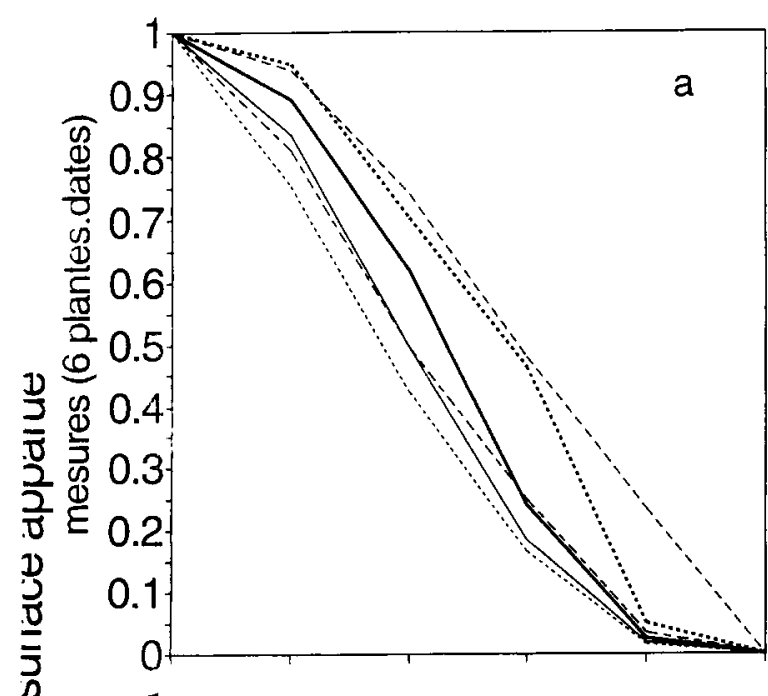

\section{Évolution des surfaces ligulées et non ligulées au cours du temps}

La figure 5 représente l'évolution des surfaces de feuilles ligulées, non ligulées visibles et de la surface totale en valeurs mesurées pour une moyenne de six plantes. Elle montre l'importance de la surface des feuilles du cornet au milieu de période d'apparition des feuilles. C'est la période où les feuilles en croissance sont les plus nombreuses et les plus grandes (fig $3 a$ ). Pour ce génotype, qui a environ 16 feuilles au total (cf Matériels et méthodes), le cornet a alors environ quatre feuilles en croissance pour sept à dix feuilles ligulées (cf Vincourt et al, 1984).

La figure 6 représente, pour chacune des méthodes (mesure et estimations), l'évolution de la moyenne des surfaces des feuilles du cornet (de six plantes) et de la différence entre la valeur mesurée et les valeurs estimées.

Les méthodes M2a et M3, peu différentes entre elles, ne s'écartent jamais beaucoup de la méthode M0. Toutes les méthodes d'estimation atténuent les pics importants de surface du cornet, observés sur toutes les plantes (fig $3 b$ ), et liés au caractère discontinu des apparitions de pointes et de ligules de feuilles.

Toutes les méthodes (sauf M2b) surestiment la surface des feuilles en croissance en début de cycle et les écarts s'inversent au milieu de la période de mise en place des feuilles. Les surestimations les plus importantes sont celles de la méthode $M 1$. La méthode $M 2 b$, satisfaisante en début de cycle, sous-estime rapidement la surface foliaire, donc ne peut être utile pour des estimations précoces.

À la fin de la période d'apparition, les méthodes M2a et M3 sous-estiment assez nettement les surfaces. Les résultats de la figure 6 sont confirmés de façon chiffrée par le calcul des écarts moyens entre mesure et estimations (RMSE, tableau I) : l'écart moyen est un peu plus fort pour M2b que pour les autres méthodes.

\section{Comparaisons des évaluations du rayonnement capté par un couvert obtenues à partir des différentes estimations de surface} nette pour la méthode $M 1$, qui est peu satisfaisante lorsque les feuilles en croissance ont des tailles très variables : le remplacement de plusieurs fractions de feuilles par une seule feuille entière n'est correcte que lorsque les tailles de feuilles sont peu variables, donc à la période où apparaissent les feuilles les plus grandes (cf figure $3 a$ ).

Le but de ces estimations de surface foliaire est de calculer le rayonnement intercepté par la culture : la qualité de l'estimation du rayonnement intercepté sera le critère de comparaison des méthodes. Elle sera jugée d'abord avec le même 

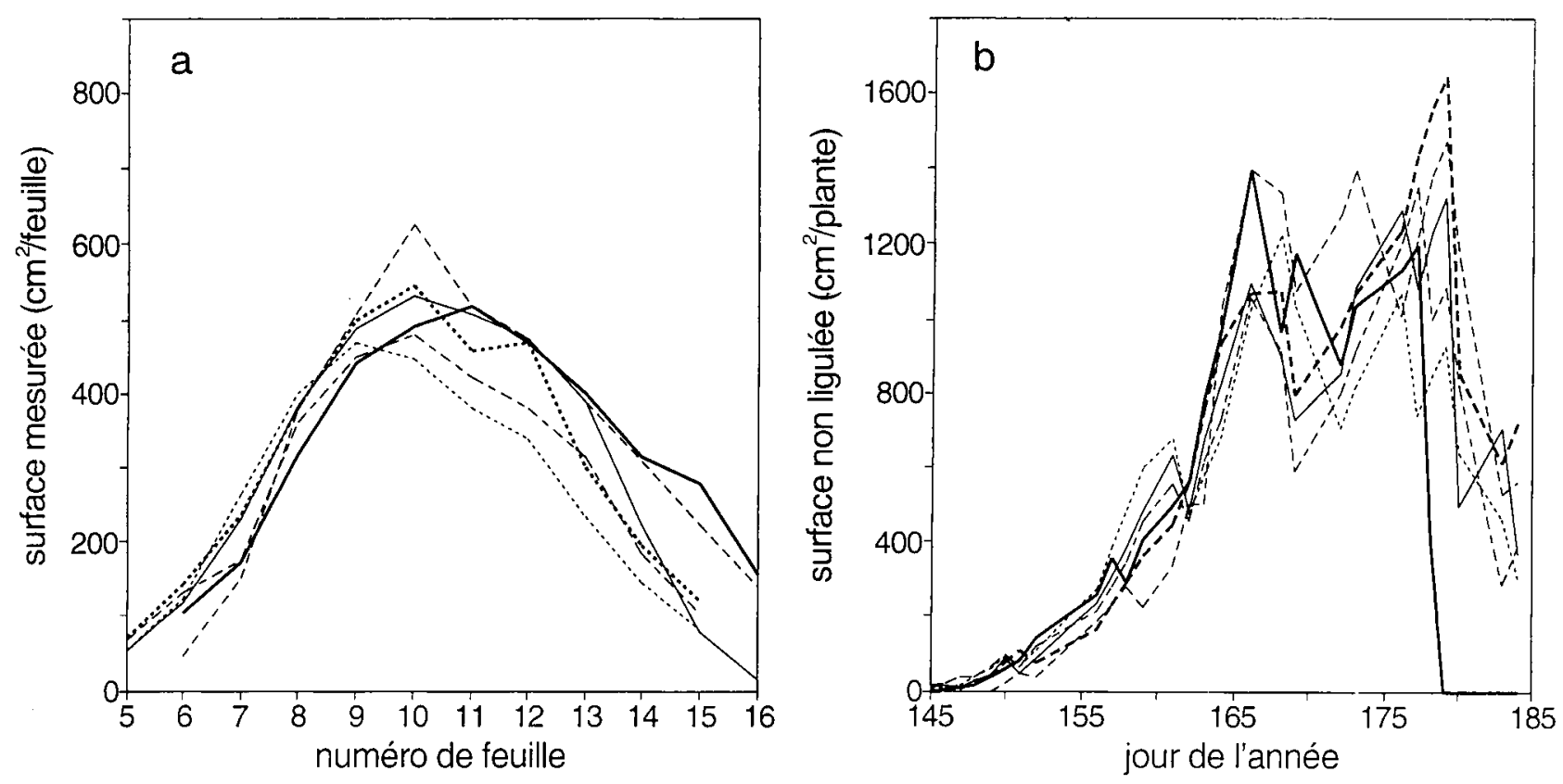

Fig 3. a. Profil de surface finale des feuilles en fonction de leur numéro, pour six plantes. Les feuilles sont numérotées à partir de la base de la plante. Les types de traits distinguent les individus. b. Évolution de la surface des feuilles non ligulées pour six plantes. Les types de traits distinguent les individus.

critère que les surfaces foliaires sur les écarts quotidiens entre mesure et estimation de rayonnement intercepté sur la période de mise en place des feuilles (tableau I), puis de façon plus globale sur l'erreur commise sur la somme de rayonnement intercepté pendant la mise en place des feuilles (tableau II).

Les écarts moyens sur les rayonnements interceptés chaque jour (tableau I) modifient le classement des méthodes mis en évidence sur les surfaces foliaires : la méthode M1 conduit à des écarts moyens plus grands que les autres méthodes : en moyenne, plus de 2 MJ PAR. $\mathrm{m}^{-2}$ d'erreur pour une moyenne quotidienne de 12. La méthode M2b estime mieux l'interception que M1, bien qu'elle estime moins bien les surfaces, car ses mauvaises estimations se situent à une période où le cornet de feuilles ne joue pas un rôle d'interception important.

Dans le tableau II, les rayonnements interceptés pendant deux périodes (jusqu'au milieu et à la fin d'apparition des feuilles) sont estimés soit à

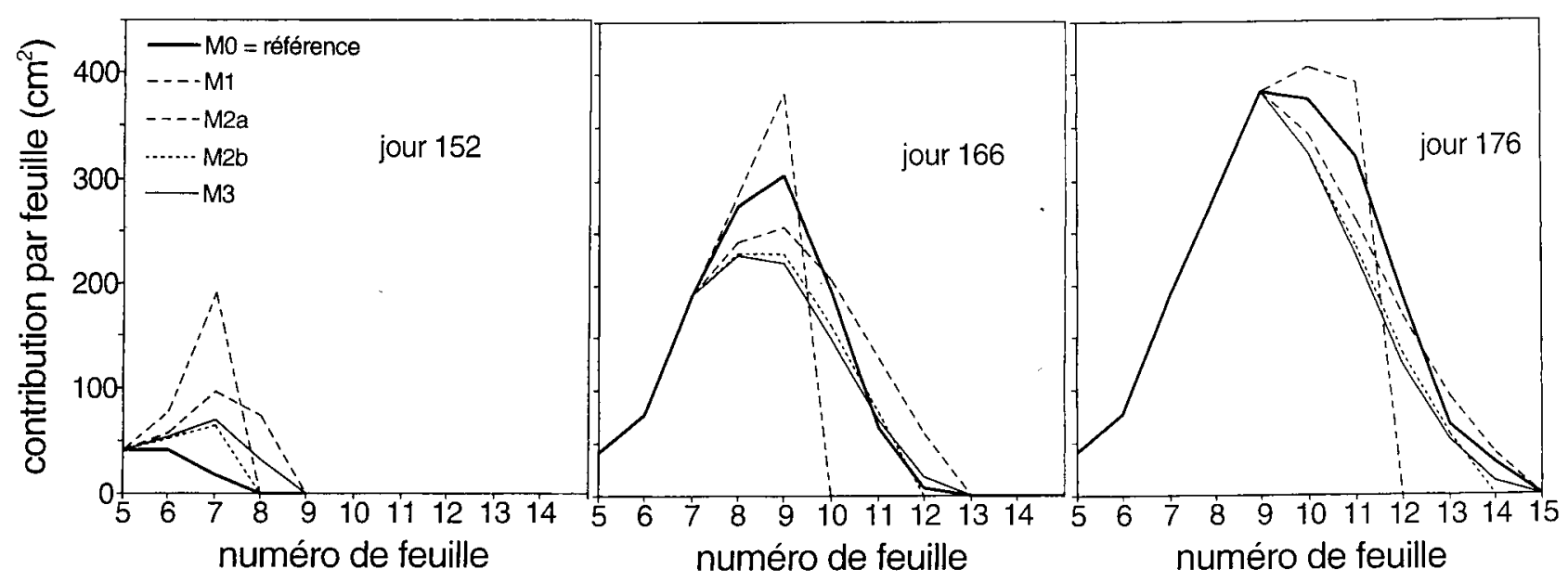

Fig 4. Surface apparue de chaque feuille pour une plante donnée et trois dates (jours 152, 166 et 176) pendant la mise en place des feuilles : mesure par éléments (M0, trait gras continu), correspondant donc à la méthode de référence; estimation par la méthode des deux prochaines feuilles ligulées (M1, tirets fins) ; estimation par la méthode des coefficients dépendant du nombre de feuilles en expansion (M2 a et $b$, pointillés fins respectivement grands et petits); estimation par la méthode par intégration de la largeur sur la longueur (M3, trait fin continu). 


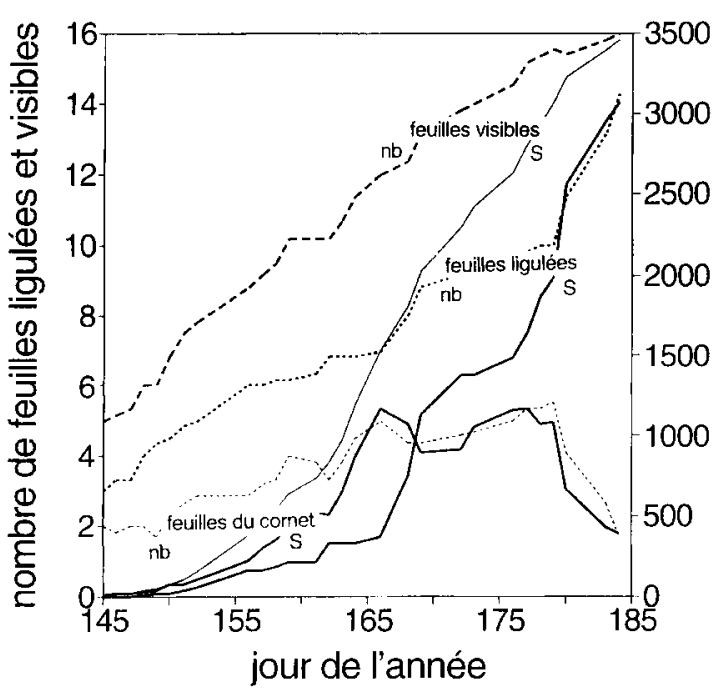

Fig 5. Évolution des nombres de feuilles (traits pointillés). Le nombre de feuilles totales visibles est en trait pointillé long, le nombre de feuilles ligulées en trait gras court, le nombre de feuilles du cornet en trait fin court. L'évolution des surfaces est en trait continu, les surfaces ligulées (courbe constamment croissante) et non ligulées (courbe croissante puis décroissante) sont en traits gras et la surface totale est en trait fin. II s'agit de la moyenne des mesures des six plantes faites selon la méthode de référence.

Tableau I. Racines des carrés moyens des écarts et écart/moyenne entre mesure et estimation pour la surface foliaire et le rayonnement intercepté (MJ

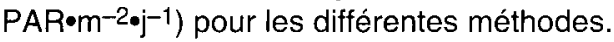

\section{$\begin{array}{llll}M 1 & M 2 a & M 2 b & M 3\end{array}$}

$\begin{array}{lrrrr}\begin{array}{l}\text { Surface foliaire } \\ \text { Écart }\left(\mathrm{cm}^{2}\right)\end{array} & 198,0 & 183,7 & 268,1 & 175,8 \\ \text { Écart/moyenne (\%) } & 36,5 & 33,8 & 59,9 & 32,4 \\ & & & & \\ & & & & \\ \text { Rayonnement intercepté } & & 1,34 & 1,87 & 1,14 \\ \left.\text { Écart (cm }{ }^{2}\right) & 2,25 & 1,3,0 & 21,0 & 12,8\end{array}$

partir des seules feuilles ligulées, c'est-à-dire en négligeant le cornet foliaire, soit à partir des surfaces mesurées ou estimées par les différentes méthodes présentées dans cet article.

Au milieu de la phase d'apparition des feuilles, le cornet foliaire est responsable de plus de $60 \%$ de l'interception de rayonnement depuis le début de la culture, d'où la nécessité d'estimer sa surface. La méthode M1 surestime fortement ( $80 \%)$ le rayonnement capté en début de cycle, ce qui s'explique par la forte surestimation de surface à cette période (fig 6). Les méthodes M2 et M3 conduisent à des erreurs d'estimations non négligeables, ce qui incite à la prudence lors de l'utilisation de ces méthodes d'estimation de surface

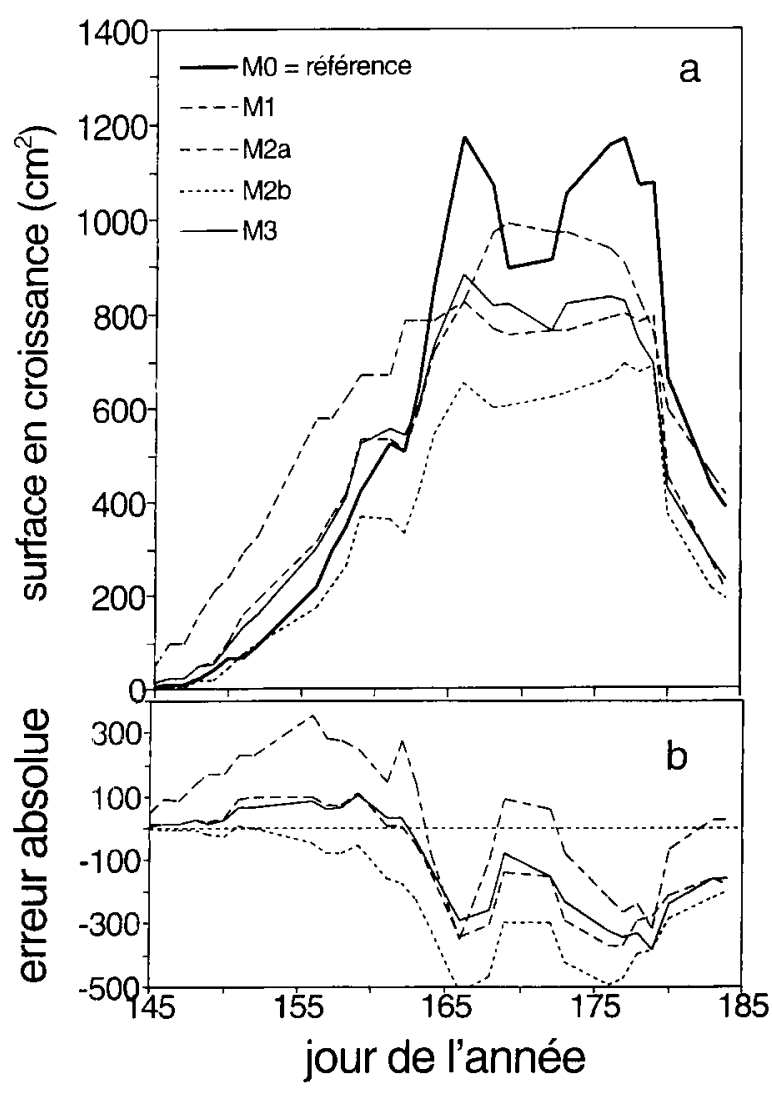

Fig 6. a. Évolution comparée des surfaces foliaires non ligulées : en trait continu gras, la référence ( $\mathrm{MO}$ ) ; en traits fins, les estimations, les tirets alternés (longs et courts) fins représentent la méthode des deux prochaines feuilles ligulées (M1) ; les pointillés fins (respectivement grands et petits), la méthode du calcul par attribution d'un coefficient à chaque feuille pour estimer la proportion de surface apparue (M2 a et b) ; le trait continu fin, la méthode par intégration de la largeur sur la longueur de la feuille (M3). Moyenne de six plantes. b. Évolution des écarts entre valeurs estimées et mesurées (moyenne des 6 plantes). Même signification des types de traits.

pour calculer des rayonnements interceptés et des efficiences de conversion en début de cycle.

À la fin de la mise en place des feuilles, c'està-dire à la floraison, le cornet foliaire n'est plus responsable que de $35 \%$ de l'interception de l'ensemble de la période semis-floraison. Le fait de le négliger peut conduire à une surestimation de $25 \%$ de l'efficience de conversion du rayonnement. Cela pourrait expliquer les fortes efficiences de conversion présentées par Bonhomme et al (1982) avant la floraison et une partie des différences entre les efficiences moyennes du semis à la floraison et du semis à la maturité. Les méthodes qui attribuent une surface à la dernière feuille apparue (M2a et M3) fournissent des estimations presque satisfaisantes : moins de $2 \%$ de différence entre la mesure et les estimations. La méthode $M 1$ surestime encore le rayonnement intercepté de près de $10 \%$. 
Tableau II. Somme de rayonnement intercepté calculée à partir de la mesure et des différentes estimations de surface foliaire et pourcentage de différence entre mesure et estimation.

\begin{tabular}{|c|c|c|c|c|}
\hline & \multicolumn{2}{|c|}{ Jour $160=$ milieu d'apparition des feuilles } & \multicolumn{2}{|c|}{ Jour $180=$ fin d'apparition des feuilles } \\
\hline & $\begin{array}{l}\text { Somme de } \\
\text { rayonnement intercepté }\end{array}$ & $\begin{array}{l}\text { Différence par rapport } \\
\quad \text { à } S L_{\text {réf }}+M O(\%)\end{array}$ & $\begin{array}{l}\text { Somme de } \\
\text { rayonnement intercepté }\end{array}$ & $\begin{array}{l}\text { Différence par rapport } \\
\text { à } S L_{\text {réf }}+M O(\%)\end{array}$ \\
\hline $\mathrm{SL}_{\text {réf }}$ & 14,7 & $-62,7$ & 189 & $-35,8$ \\
\hline $\mathrm{SL}$ & 15,2 & $-61,5$ & 192 & $-34,7$ \\
\hline $\mathrm{SL}_{\text {réf }}+\mathrm{MO}$ & 39,5 & 0,0 & 294 & 0,0 \\
\hline $\mathrm{SL}+\mathrm{M} 1$ & 70,8 & 79,1 & 320 & 8,9 \\
\hline $\mathrm{SL}+\mathrm{M} 2 \mathrm{a}$ & 48,6 & 22,6 & 289 & $-1,7$ \\
\hline $\mathrm{SL}+\mathrm{M} 2 \mathrm{~b}$ & 34,9 & $-11,8$ & 265 & $-9,9$ \\
\hline $\mathrm{SL}+\mathrm{M} 3$ & 47,1 & 19,1 & 290 & $-1,5$ \\
\hline
\end{tabular}

L'amélioration des estimations de rayonnement intercepté lorsqu'on allonge la période de calcul provient du fait que les quantités interceptées en début de cycle sont faibles rapportées à celles de l'ensemble du cycle : les erreurs commises sur les surfaces en début de cycle ont donc peu d'influence sur la valeur de la somme de rayonnement intercepté sur l'ensemble du cycle.

\section{CONCLUSION}

La possibilité d'estimer la surface maximale des feuilles à partir des longueurs et largeurs est confirmée, ainsi que la stabilité du coefficient de forme $(0,75)$.

L'accroissement en surface de chaque feuille du cornet (M2) peut être simulé de façon satisfaisante à partir de sa position relative dans le cornet et de sa surface finale. Le fait de simuler un accroissement en longueur et d'utiliser une loi reliant longueur et largeur le long de la feuille (M3) donne une estimation de surface des feuilles en croissance de qualité analogue.

Du point de vue du calcul du rayonnement intercepté, les méthodes $\mathrm{M} 2$ et $\mathrm{M} 3$ ont des performances analogues, mais M2 sera choisie de préférence à cause de sa simplicité d'utilisation. Elle permet de passer de l'estimation de l'indice foliaire maximal, obtenu à partir des dimensions maximales des feuilles, à l'estimation de son évolution, en ne faisant comme observation supplémentaire que des comptages de feuilles. Le choix entre $\mathrm{M} 2 \mathrm{a}$ et $\mathrm{M} 2 \mathrm{~b}$ dépend de sa période d'utilisation : M2b est préférable seulement si l'on s'intéresse à des interceptions de rayonnement en début de cycle (première moitié de la période semis-floraison). Pour des estimations sur des périodes plus longues, M2a est plus satisfaisante.

L'emploi généralisé de la méthode M2a nécessiterait confirmation sur d'autres génotypes de maîs, mais son test dans d'autres conditions pose le problème de la lourdeur de la méthode de référence pour mesurer l'allongement quotidien des feuilles. Elle pourrait être décomposée en confirmation de la loi de forme des feuilles et la loi d'allongement des feuilles du cornet, utilisant la proportionnalité entre surface apparue et position de la feuille dans le cornet.

La mesure des surfaces foliaires (sur maïs ou autres graminées) pourrait encore être simplifiée en utilisant les relations entre nombre de feuilles ligulées et non ligulées mises en évidence par Vincourt (1984), puis des estimations de surfaces foliaires individuelles à partir de la surface de la plus grande feuille.

\section{RÉFÉRENCES}

Beardsell MF (1977) Effects of routine handling on maize growth. Aust J Plant Physiol 4, 857-861

Bonhomme R, Varlet-Grancher C (1978) Estimation of the gramineous crop geometry by plant profiles including leaf width variations. Photosynthetica 12, 2, 193-196

Bonhomme R, Ruget F, Derieux M, Vincourt P (1982) Relations entre production de matière sèche et énergie interceptée chez différents génotypes de maïs. C R Acad Sc Paris, sér III 294, 393-398

Chartier P, Perrier A, Verbrugghe M (1971) Assimilation nette, utilisation de l'eau et microclimatologie d'un champ de maïs. I. Objectifs et présen- 
tation du dispositif expérimental de La Minière. Ann Agron 22, 367-381

Dwyer LM, Stewart DW (1986) Leaf area development in field-grown maize. Agron J 78, 334-343

Edmeades GO, Daynard TB (1979) The relationship between final grain yield and photosynthesis at flowering in individual maize plants. Can J Plant Sci $59,585-601$

Monteith JL (1972) Solar radiation and productivity in tropical ecosystems. J App/ Eco/ 9, 747-766

Muchow RC, Carberry PS (1989) Environmental control of phenology and leaf growth in a tropically adapted maize. Field Crops Res 20, 221-236
Muchow RC, Sinclair TR, Bennett JM (1990) Temperature and solar radiation effects on potential maize yield across locations. Agron $J$ 82, 338343

Prévot L, Aries F, Monestiez P (1991) Modélisation de la structure géométrique du maïs. agronomie 11 , 491-503

Sanderson JB, Daynard TB, Tollenaar M (1981) A mathematical model of the shape of corn leaves. Can J Plant Sci 61, 109-1011

Vincourt $P$ (1984) Relations entre rythme d'apparition des feuilles, nombre total de feuilles et précocité de floraison chez le maïs. agronomie 4, 795-800 